\title{
Quantum simulation of Dirac fermion mode, Majorana fermion mode and Majorana-Weyl fermion mode in cavity QED lattice
}

\author{
Sujit Sarkar \\ Poornaprajna Institute of Scientific Research, \\ 4 Sadashivanagar, Bangalore 5600 80, India. \\ e-mail: sujit.tifr@gmail.com
}

(Dated: August 22, 2018)

\begin{abstract}
Quantum simulation aims to simulate a quantum system using a controble laboratory system that underline the same mathematical model. Cavity QED lattice system is that prescribe system to simulate the relativistic quantum effect. We quantum simulate the Dirac fermion mode, Majorana fermion mode and Majorana-Weyl fermion mode and a crossover between them in cavity QED lattice. We also present the different analytical relations between the field operators for different mode excitations.

PACS: 42.50.Pq, 03.65.Vf, 42.50.-p
\end{abstract}

Introduction: The difficulties in observing the real quantum relativistic effects have generated immense interest in the quantum simulation physics. In recent years, there has been increased interest in the simulation of relativistic quantum effects using different physical system in which parameters tunibility allows access to different physical regimes [1 11]. These difficulties of observing quantum relativistic effects stimulate us to study the quantum simulation physics of Dirac fermion mode, Majorana fermion mode and Majorana-Weyl fermion modes in one dimensional cavity QED lattice and a crossover from one mode of excitations to the another using the tunibility of the physical parameters of the system.

In quantum simulation, one aim is to simulate a quantum system using a controllable laboratory system that underlines the same mathematical models. Therefore it is possible to simulate a quantum system that can be neither efficiently simulated on a classical computer nor easily accessed experimentally.

The recent experimental success in engineering strong interaction between the photons and atoms in high quality micro-cavities opens up the possibility to use the light matter system as quantum simulators for many body physics [9-29]. Many interesting results are coming out to understand the complicated quantum many body system. A focus on the coupled cavities is one of the most potential candidate for an efficient quantum simulator due to the control of the microcavities parameters and success of fabrication of large scale cavity arrays [25-26]. The concept and existence of Majorana fermion mode is one of the most advance research area in quantum condensed matter system. Majorana introduced a special kind of fermions which are their own antiparticle, i.e., the neutral particle [28, 29]. He introduced this particle to describe neutrions. In recent years, there are several candidates of Majorana fermions in quantum condensed matter system like quantum Hall system with filling fraction 5/2 30, 31]. Kitaev first found the existence of Majorana fermion mode in one dimensional model [32]. Many research groups have already proposed the physically ex- istence of MFs at the edge state of $1 \mathrm{D}$ system like electrostatic defects lines in superconductor, quasi-one dimensional superconductor and cold atom trapped in one dimension [33, 34]. Majorana fermions obey the nonAbelian statics both in 2D and 1D, allowing of certain gate operation required in quantum computation [35]. In the present study one of our goal is to predict the presence of Dirac fermion mode, Majorana fermion mode and Majoran-Weyl fermion mode and crossover in our model system. In the Majorana-Weyl fermion mode, the fermion mode satisfy the condition for particle and antiparticle condition but the excitation is massless.

There are few studies to simulate the Majorana fermion modes in cavity QED system but the model Hamiltonian and the associate relevant physics are different and at the same time there is no coossover study between the Dirac and Majorana fermion modes in the literature of cavity QED system [9-11].

The authors of Ref.3 have found the process of Dirac to Majorana fermion converator on the surface of a 3D topological insulator. A Dirac fermion injected by the voltage source is split into a pair of Majorana fermion and then finally recombine before going to drain. But in our present study there is no such split and fusion process of Dirac and Majorana fermion. In our study the system is in Dirac fermion mode when only a atom-photon coupling or a single Rabi frequency oscillation present in the system and the existence of Majorana fermions when two atom photon couplings and the two laser frequencies are simultaneously present in the system. For a quantum simulated Hamiltonian for Majorana fermion mode for a specific mathematical relation between the Rabi frequencies oscillation, the atom-photon coupling strengths and the laser field detuning.

To the best of our knowledge the quantum simulation physics for the different kind of fermionic mode in a same cavity QED system is absent in the literature.

In the present study, we simulate two model Hamiltonians through the proper tuning of Rabi frequencies and the atom-photon coupling strengths in the system which 
quantum simulate different modes of fermionic excitations.

\section{The Model Hamiltonian:}

The Hamiltonian of our present study consists of three parts:

$$
H=H_{A}+H_{C}+H_{A C}
$$

The Hamiltonians are the following

$$
H_{A}=\sum_{j=1}^{N} \omega_{e}\left|e_{j}><e_{j}\right|+\omega_{a b}\left|b_{j}><b_{j}\right|
$$

where $j$ is the cavity index. $\omega_{a b}$ and $\omega_{e}$ are the energies of the state $\mid b>$ and the excited state respectively. The energy level of state $\mid a>$ is set as zero. $\mid a>$ and $\mid b>$ are the two stable state of a atom in the cavity and $\mid e>$ is the excited state of that atom in the same cavity. The following Hamiltonian describes the photons in the cavity,

$$
H_{C}=\omega_{C} \sum_{j=1}^{N} a_{j}^{\dagger} a_{j}+J_{C} \sum_{j=1}^{N}\left(a_{j}^{\dagger} a_{j+1}+h . c\right)
$$

where $a_{j}^{\dagger}\left(a_{j}\right)$ is the photon creation (annihilation) operator for the photon field in the $j$ 'th cavity, $\omega_{C}$ is the energy of photons and $J_{C}$ is the tunneling rate of photons between neighboring cavities. The interaction between the atoms and photons and also by the driving lasers are described by

$$
H_{A C}=\sum_{j=1}^{N}\left[\left(\frac{\Omega_{a}}{2} e^{-i \omega_{a} t}+g_{a} a_{j}\right)\left|e_{j}><a_{j}\right|+h . c\right]+[a \leftrightarrow b] .
$$

Here $g_{a}$ and $g_{b}$ are the couplings of the cavity mode for the transition from the energy states $\mid a>$ and $\mid b>$ to the excited state. $\Omega_{a}$ and $\Omega_{b}$ are the Rabi frequencies of the lasers with frequencies $\omega_{a}$ and $\omega_{b}$ respectively.

The authors of Ref. [14-16] have derived an effective spin model by considering the following physical processes: A virtual process regarding the emission and absorption of photons between the two stable states of neighboring cavity yields the resulting effective Hamiltonian as

$$
H_{x y}=\sum_{j=1}^{N} B{\sigma_{j}}^{z}+\sum_{j=1}^{N}\left(\frac{J_{1}}{2} \sigma_{j}{ }^{\dagger} \sigma_{j+1}{ }^{-}+\frac{J_{2}}{2}{\sigma_{j}}^{-}{ }^{-}{ }_{j+1}{ }^{-}+h . c\right)
$$

When $J_{2}$ is real then this Hamiltonian reduces to the $\mathrm{XY}$ model. Where $\sigma_{j}{ }^{z}=\left|b_{j}><b_{j}\right|-\left|a_{j}><a_{j}\right|$, $\sigma_{j}{ }^{+}=\left|b_{j}><a_{j}\right|, \sigma_{j}^{-}=\left|a_{j}><b_{j}\right|$.

$$
H_{x y}=\sum_{i=1}^{N} B\left(\sigma_{i}^{z}+J_{x} \sigma_{i}^{x} \sigma_{i+1}^{x}+J_{y} \sigma_{i}^{y} \sigma_{i+1}{ }^{y}\right) .
$$

With $J_{x}=\left(J_{1}+J_{2}\right)$ and $J_{y}=\left(J_{1}-J_{2}\right)$.

We follow the references [14, 36], to present the analytical expression for the different physical parameters of the system.

$B=\frac{\delta_{1}}{2}-\beta, \beta$ is define in Ref. [37].

$$
J_{1}=\frac{\gamma_{2}}{4}\left(\frac{\left|\Omega_{a}\right|^{2} g_{b}{ }^{2}}{\Delta_{a}{ }^{2}}+\frac{\left|\Omega_{b}\right|^{2} g_{a}{ }^{2}}{\Delta_{b}{ }^{2}}\right), J_{2}=\frac{\gamma_{2}}{2}\left(\frac{\Omega_{a} \Omega_{b} g_{a} g_{b}}{\Delta_{a} \Delta_{b}}\right) .
$$

Where $\gamma_{a, b}=\frac{1}{N} \sum_{k} \frac{1}{\omega_{a, b}-\omega_{k}} \gamma_{1}=\frac{1}{N} \sum_{k} \frac{1}{\left(\omega_{a}+\omega_{b}\right) / 2-\omega_{k}}$ and $\gamma_{2}=\frac{1}{N} \sum_{k} \frac{e^{i k}}{\left(\omega_{a}+\omega_{b}\right) / 2-\omega_{k}} \delta_{1}=\omega_{a b}-\left(\omega_{a}-\omega_{b}\right) / 2$, $\Delta_{a}=\omega_{e}-\omega_{a} . \Delta_{b}=\omega_{e}-\omega_{a}-\left(\omega_{a b}-\delta_{1}\right) . \delta_{a}{ }^{k}=\omega_{e}-\omega_{k}$, $\delta_{b}{ }^{k}=\omega_{e}-\omega_{k}-\left(\omega_{a b}-\delta_{1}\right), \omega_{k}=\omega_{c}+J_{c} \sum_{k} \cos k . g_{a}$ and $g_{b}$ are the couplings of respective transition to the cavity mode, $\Omega_{a}$ and $\Omega_{b}$ are the Rabi frequency of laser with frequency $\omega_{a}$ and $\omega_{b}$.

Quantum Simulation for Dirac Fermion mode and Mathematical Relation Between the Fields : Here we quantum simulate the Dirac fermion physics through the proper tuning of cavity QED lattice parameters. This condition can be achieve when $J_{x}=J_{y}$. This condition implies that $2 J_{2}=0$. It is clear from the analytical expression of $J_{1}$ and $J_{2}$ that to satisfy the condition one of the atom-photon coupling strength, i.e., $g_{a}$ or $g_{b}$ be zero or one of the Rabi frequency oscillation $\Omega_{a}$ or $\Omega_{b}$ should be zero. The analytical expression for $J_{1}$ become $J_{1}=\frac{\gamma_{2}}{4} \frac{\left|\Omega_{a}\right|^{2} g_{b}{ }^{2}}{\Delta_{a}{ }^{2}}$ or $J_{1}=\frac{\gamma_{2}}{4} \frac{\left|\Omega_{b}\right|^{2} g_{a}{ }^{2}}{\Delta_{b}{ }^{2}}$. The other condition is $\delta_{1}=2 \beta$, i.e., $2 \omega_{a b}-\left(\omega_{a}-\omega_{b}\right)=4 \beta$. Therefore the condition for quantum simulation relates upto the microscopic level.

In this limit the Hamiltonian reduced to

$$
H=J \sum_{i=1}^{N}\left(\sigma_{i}^{x} \sigma_{i+1}^{x}+\sigma_{i}^{y} \sigma_{i+1}^{y}\right)
$$

After the Jordan-Wigner transformation and Abelian Bosonization study one can write the above Hamiltonian as [41, 43]

$$
H_{0}=\sum_{s} \int \frac{d k}{2 \pi} \epsilon(k) \psi_{s}^{\dagger}(k) \psi_{s}(k)
$$

$H_{0}=\sum_{s} \int_{-\Lambda}^{\Lambda} \frac{d k}{2 \pi}\left(k v_{F}\right)\left(\psi_{s, R}^{\dagger}(k) \psi_{s, R}(k)-\psi_{s, L}^{\dagger}(k) \psi_{s, L}(k)\right)$

Here we use $\epsilon_{k}=k v_{F}$ near the Fermi points and $\psi_{s}^{\dagger}(x)=\left(\psi_{s, R^{\dagger}}(x), \psi_{s, L^{\dagger}}(x)\right)$. Following the discussions in addendum, we can write the above Hamiltonian in the following form of Dirac equation without any mass term.

$$
H=2 J \int d x \bar{\psi}\left(i \gamma_{1}\right) \partial_{x} \psi
$$


and $\psi^{\dagger}(x)=\left(\psi_{R}^{\dagger}, \psi_{L}^{\dagger}\right) \cdot \bar{\psi}^{\dagger}(x)=\left(\psi_{L}^{\dagger}, \psi_{R}^{\dagger}\right)$, $\psi_{s}(x)=\int \frac{d k}{2 \pi} \psi_{s}(k) e^{i k x}$. It is customery to introduce the Dirac matrices in Dirac equation. The $\gamma$ matrices are the following. $\gamma^{0}=\left(\begin{array}{ll}0 & 1 \\ 1 & 0\end{array}\right), \gamma^{1}=-i \sigma_{y}=\left(\begin{array}{cc}0 & -1 \\ 1 & 0\end{array}\right)$, $\gamma^{5}=\gamma_{0} \gamma_{1}=\sigma_{z}=\left(\begin{array}{cc}1 & 0 \\ 0 & -1\end{array}\right)$,

$\psi_{R}$ and $\psi_{L}$ are the fermionic field for the right and left movers electron. The scalar field and its dual can be expressed as $\phi(x)=\phi_{R}(x)+\phi_{L}(x), \theta(x)=-\phi_{R}(x)+\phi_{L}(x)$. The detail derivation of the analytical relation between the wave function and the scalar field are relegated to the addendum. Here we only present the final form Now we present the mathematical relation between the operators, $e^{ \pm \phi(x)}$ and $e^{ \pm \theta(x)}$.

$e^{i \alpha \sqrt{\pi} \phi(x 1)} e^{i \beta \sqrt{\pi} \theta(x 2)}=e^{i \beta \sqrt{\pi} \theta(x 2)} e^{i \alpha \sqrt{\pi} \phi(x 1)} \operatorname{sign}(x 2-x 1)$

$e^{i \alpha \sqrt{\pi} \phi(x 1)} \psi_{R / L}(x 2)=\psi_{R / L}(x 2) e^{i \alpha \alpha \sqrt{\pi} \phi(x 1)} \operatorname{sign}(x 2-x 1)$

$e^{i \beta \sqrt{\pi} \theta(x 1)} \psi_{R / L}(x 2)=-\psi_{R / L}(x 2) e^{i \beta \sqrt{\pi} \theta(x 1)} \operatorname{sign}(x 2-x 1)$.

Therefore it is clear from our theoretical analysis that the cavity QED lattice shows the Dirac fermion like mode when one of the atom-photon coupling $\left(g_{a}\right.$ or $\left.g_{b}\right)$ is zero or one of the applied Rabi frequencies $\left(\Omega_{a}, \Omega_{b}\right)$ is zero.

Dirac Equation for Majorana Fermion mode and Condition of Majorana-Weyl fermionic mode: Here we quantum simulate the Majorana fermion mode and the Majorana-Weyl fermion mode. Majorana thought whether it might be possible for spin-1/2 particle to be its own anti-particle. To get an equation alike to Dirac equation but capable of governing a real field that requires the $\gamma$ matrices of that equation must satisfy the Clifford algebra are purely imaginary. Here we show explicitly that the $\gamma$ matrices of Majorana equation are purely imaginary and field satisfy the particleantiparticle equivalent condition.

We also derive the condition for the excitation of Majorana-Weyl fermion mode where the simulated mode shows the gapless excitation.

We consider $J_{1}=J_{2}, J_{x}$ become $J_{1}+J_{2}$ and $J_{y}=0$. In the microcavity array, the condition for $J_{1}=J_{2}$ achieve when

$$
\Omega_{a}^{2} g_{b}^{2} \Delta_{b}^{2}+\Omega_{b}^{2} g_{a}^{2} \Delta_{a}^{2}=2 \Omega_{a} \Omega_{b} g_{a} g_{b} \Delta_{a} \Delta_{b} .
$$

The above condition implies that $\Omega_{a}=\Omega_{b} \frac{g_{a} \Delta_{a}}{g_{b} \Delta_{b}}$. The only constraint is that $\Delta_{a} \neq \Delta_{b}$, the magnetic field diverge when $\Delta_{a}=\Delta_{b}$. At the same time, $\Omega_{a}=\Omega_{b}$ and $g_{a}=$ $g_{b}$ are also not possible because this limit also leads to the condition $\Delta_{a}=\Delta_{b}$. Suppose we consider, $\Omega_{a}=$ $\alpha_{1} \Omega_{b}, g_{a}=\alpha_{2} g_{b}$ and $\Delta_{a}=\alpha_{3} \Delta_{b}$. These relations implies that $\alpha_{1}{ }^{2}+\alpha_{2}^{2} \alpha_{3}{ }^{2}=2 \alpha_{1} \alpha_{2} \alpha_{3} . \alpha_{1}=\alpha_{2} \alpha_{3}, \alpha_{1}, \alpha_{2}$ and $\alpha_{3}$ are the numbers. These analytical relations help to implement the transverse Ising model Hamiltonian but $\alpha_{1}, \alpha_{2}$ and $\alpha_{3}$ should not be equal to 1 .

The quantum state engineering of cavity QED is in the state of art due to the rapid progress of technological development of this field [1]. Therefore one can achieve this limit to get the desire quantum state. One can write the final Hamiltonian as,

$$
H_{T}=B \sum_{j=1}^{N}\left(\sigma_{z}(j)+\lambda \sigma_{x}(j) \sigma_{x}(j+1)\right),
$$

where $\lambda=\frac{J_{1}+J_{2}}{B}$. The effective Hamiltonian become the transverse Ising model which studied in the previous literature [38 40].

One can also write the starting Hamiltonian as through the rotation in spin basis

$$
H=\sum_{n}\left[\lambda \sigma_{z}(n) \sigma_{z}(n+1)+s_{x}(n)\right] .
$$

We recast the Hamiltonian in the following form because it will help us to use the order and disorder operator directly to the derivation of the equation of motion and finally the Dirac equation for Majorana fermion.

Here our main motivation is to use some of important results of this model Hamiltonian to discuss the relevant physics of array of cavity QED system. We introduce the order and disorder operator in the addendum [39, 40]. These operators are defining the sites of the dual lattice, i.e., we define the operator between the nearest-neighbor site of the original lattice. Here we define the Dirac spinor, $\chi_{1}(n)=\sigma_{z}(n) \mu_{z}(n+1 / 2)$ and $\chi_{2}(n)=\sigma_{z}(n) \mu_{z}(n-1 / 2)$.

Now our main task is to find the equation of motion for the operators, $\sigma_{3}(n)$ and $\mu_{3}(n)$ which help us to build the Dirac equation. The detail derivation are relagated to the appendix.

The equation of motion for the $\sigma_{z}(n)$ is the following:

$$
\frac{\partial \sigma_{z}(n)}{\partial \tau}=\left[H, \sigma_{z}(n)\right]=\sigma_{x}(n) \sigma_{z}(n)
$$

The equation of motion for $\mu_{z}(n+1 / 2)$ is the following:

$$
\begin{aligned}
\frac{\partial \mu_{z}(n+1 / 2)}{\partial \tau}= & \lambda \mu_{x}(n+1 / 2) \mu_{z}(n+1 / 2) \\
& \left.=\lambda \sigma_{z}(n) \sigma_{z}(n+1 / 2) \mu_{z}(n+1 / 2) 19\right)
\end{aligned}
$$

$$
\begin{aligned}
& \frac{\partial \chi_{1}(n)}{d \tau}=-\chi_{2}(n)+\lambda \chi_{2}(n+1) . \\
& \frac{\partial \chi_{2}(n)}{d \tau}=-\chi_{1}(n)+\lambda \chi_{1}(n-1) .
\end{aligned}
$$


These two fields, $\chi_{1}(n)$ and $\chi_{2}(n)$ satisfy the following relations, $\left\{\chi_{1}(n 1), \chi_{2}(n 2)\right\}=2 \delta_{n 1, n 2}$. One can write down the above equation in the following compact form,

$$
\left(\gamma^{0} \frac{\partial}{\partial t}+\gamma^{3} \frac{\partial}{\partial r}+m\right) \chi=0
$$

where $\chi^{\dagger}=\left(\chi_{1}, \chi_{2}\right)$ and $m=\frac{1-\lambda}{\alpha}, \gamma^{0}=\left(\begin{array}{ll}0 & 1 \\ 1 & 0\end{array}\right), \gamma^{3}=$ $\left(\begin{array}{cc}1 & 0 \\ 0 & -1\end{array}\right)$.

One can also write the above Majorana equation in a compact form:

$$
\left(i \tilde{\gamma}^{\mu} \partial_{\mu}-m\right) \chi=0
$$

Where $\tilde{\gamma}^{0}=\left(\begin{array}{cc}0 & i \\ i & 0\end{array}\right), \tilde{\gamma}^{3}=\left(\begin{array}{cc}i & 0 \\ 0 & -i\end{array}\right)$.

Therefore we prove that the spinor field satisfy the Majorana condition of spin-1/2 particle and also the $\tilde{\gamma}$ matrices are imaginary.

The condition for the massless Majorana fermion field is that $m=0$, i.e., $\lambda=1$. In this quantum simulation process, one can get through this massless excitation through this analytical relation.

$\frac{\gamma_{2}}{2}\left(\frac{\left|\Omega_{a}\right|^{2} g_{b}{ }^{2}}{\Delta_{a}{ }^{2}}+\frac{\left|\Omega_{b}\right|^{2} g_{a}{ }^{2}}{\Delta_{b}{ }^{2}}\right)=\frac{\omega_{a b}}{2}-\frac{1}{4}\left(\omega_{a}-\omega_{b}\right)-\beta$.

We term this fermionic mode as Majorana-Weyl fermionic mode. In this study we obtain the different kind of fermionic modes from the quantum simulation of the system, here there is no splitting of Dirac fermion mode into the Majorana fermion mode or MajoranaWeyl fermionic mode. There are no studies in the previous literature of cavity QED where one has found a crossover from Dirac fermion modes to Majorana fermion modes [9-11].

Here we present the analytical relations between the Majorana fermion operators with the order and disorder operator with the free Dirac field in Abelian bosonization theory. Then one obtains the following sets of commutation relations.

$$
\begin{gathered}
\sigma_{z}(x 1) \mu_{z}(x 2)=\mu_{z}(x 2) \sigma_{z}(x 1) \operatorname{sign}(x 1-x 2) \\
\sigma_{z}(x 1) \chi(x 2)=\chi(x 2) \sigma_{z}(x 1) \operatorname{sign}(x 1-x 2) \\
\mu_{z}(x 1) \chi(x 2)=-\chi(x 2) \sigma_{z}(x 1) \operatorname{sign}(x 1-x 2)
\end{gathered}
$$

It is very clear from the above analytical relations that $\chi_{1}^{\dagger}=\chi_{1}$ and $\chi_{2}^{\dagger}=\chi_{2}$. The detail derivation is relegated in the appendix. The above relation has similarity with the free Dirac field in Abalian bosonization theory, where Dirac field operator is a local product of two phase exponential depending on the scalar field and its dual [4-43], as one study the Luttinger liquid physics in Abelian bosonization theory.

\section{Conclusions}

We have presented the existence of Dirac fermion mode, Majorana fermion mode and also Majorana-Weyl fermionic mode for the optical cavity array with the relation between Rabi frequency oscillation and the atom photon coupling strength. We have also presented the crossover between the Dirac fermion modes to Majorana fermion modes and also the condition for appearence of Majorana-Weyl massless excitation mode. We have also presented several analytical relations between the Majorana field, order and disorder operators.

Acknowledgement: The author would like to acknowledge the discussions with Prof. S. Girvin during the international workshop/school on Dirac Materials and Chandrashekar lecture at ICTS and the library and LAMP group of Raman Research Institute. The author would like to thank Dr. P. K. Mukherjee for reading the manuscript carefully. Finally the author would like to acknowledge the DST (SERC) project and HRI Library.
[1] R. Gerritsma et al., Nature 463, 68 (2010).

[2] Kangjun Seo, Li Han and C. A. R. Sa de Melo, Phys. Rev. Lett 109, 105303 (2012).

[3] Liang Fu and C. L. Kane, Phys. Rev. Lett (2009).

[4] L. Lamta, J. Leo'n, T. Schatz and E. Solano, Phys. Rev. Lett (2007).

[5] A. Bermudez, M. A. Martin-Delgado and E. Solano, Phys. Rev. A 76041801 (2007).

[6] , X. Zhang, Phys. Rev. Lett 100, 113903 (2008).

[7] J. Otterbach, R. G. Unanyan and M. Fleishhauer, Phys. Rev. Lett 102, 0636602 (2008).

[8] J. Y. Vaishanav and C. W. Clark, Phys. Rev. Lett 100, 153002 (2006).

[9] C. E. Bardyn and A. Imamoglu, arXiv(condmat)/1204.1238 .
[10] Brijesh Kumar and Somenath Jalal, arXiv(condmat)/1210.6922.

[11] Mircea Trif and Yaroslav Tserkovnyak, arXiv (condmat)/1202.2694.

[12] G. Agarwal, "Quantum Optics", (Cambridge University Press, Delhi 2013).

[13] Greentree. A D et al. 2006, Nature Phys. 466856.

[14] Hartmann Michael J, Fernando G S, Brando L and Plenio Martin B 2006 Nature Phys 462 849; Hartmann Michael J, Fernando G S, Brando L and Plenio Martin B 2008, Laser and Photonics Rev. 2527.

[15] Hartmann Michael J, Fernando G S, Brando L and Plenio Martin B 2007, Phys. Rev. Lett 99160501.

[16] S. Sarkar, Physica B 407, 44 (2012).

[17] A. C. Ji, X. C. Xie, and W. M. Liu , Phys. Rev.Lett. 99, 
$183602(2007)$

[18] T. Byrnes, N. Y. Kim, K. Kusudo, and Y. Yamamoto, Phys. Rev. B 78075320 (2008).

[19] I. Carusotto I et al., arXiv:0812.4195 (2008).

[20] M. J. Bhaseen, M. Hohenadler, A. O. Silver, and B. D. Simons, Phys. Rev. Lett. 102135301 (2009).

[21] J. Zhao, A. W. Sandvik and K. Ueda, arXiv:0806.3603 (2008).

[22] P. Pippan, H. G. Evertz, and M. Hohenadler , arXiv: 0904.1350 (2009).

[23] M. Aichhorn et al., Phys. Rev. Lett. 100216401 (2008).

[24] S. Schmidt, and G. Blatter, arXiv:0905.3344 (2009).

[25] M. I. Hwang and M. S. Choi, arXiv:1207.0082 (2012).

[26] D. G. Angelakis, M. F. Santos and S. Bose, Phys. Rev. A 76 R031805 (2007).

[27] S. Horoche and J. M. Raimond 2006 in Exploring the Quantum Atoms, Cavities, and Photons, (Oxford University Press).

[28] E. Majorana, Nuovo Cimento 14, 171 (1937).

[29] F. Wilczek, Majorana returns, Nature Physics 5, 614

[30] G. Moore and N. Read, Nucl. Phys. B 360, 362 (1991).

[31] N. Read and D. Green, Phys. Rev. B 61, 10267 (2000).

[32] A. Y. Kitaev, Physics-Uspekhi 44, 131 (2001).

[33] M. Wimmer et al., Phys. Rev. Lett 105, 046803 (2010).

[34] N. B. Kopnin and M. M. Salomaa, Phys. Rev. B 44, 9667 (1991).

[35] C. Nayak et al., Rev. Mod. Phys 80, 1083 (2008).

[36] F. V. James and J. Jerke, Can. J. Phys 85, 625 (2007). $[37]$

$$
\begin{aligned}
\beta= & \frac{1}{2}\left[\frac { | \Omega _ { b } | ^ { 2 } } { 4 \Delta _ { b } } \left(\Delta_{b}-\frac{\left|\Omega_{b}\right|^{2}}{4 \Delta_{b}}-\frac{\left|\Omega_{b}\right|^{2}}{4\left(\Delta_{a}-\Delta_{b}\right)}-\gamma_{b} g_{b}{ }^{2}-\gamma_{1} g_{a}{ }^{2}\right.\right. \\
& \left.+\gamma_{1}{ }^{2} \frac{g_{a}{ }^{4}}{\Delta_{b}}-(a \leftrightarrow b)\right] .
\end{aligned}
$$

[38] Subir Sachdev, Quantum Phase Transitions, Cambridge University Press, 2001.

[39] G. Mussardo, "Statistical Field Theory", Oxford Graduate Texts (New Delhi, 2010).

[40] Itzyaskon and Druff, "Statistical Field Theory", (Cambridge University Press, New York 1989).

[41] T. Giamarchi, " Quantum Physics in One Dimension".

[42] A.O. Gogolin, A. A. Nersesyan and A. M. Tsvelik, "Bosonization and Strongly Correlated Systems", Cambridge University Press (New York, 1998).

[43] E. Fradkin, " Field Theories in Condensed Matter Physics", Cambridge University Press, Delhi 2013. .

\section{Addendum}

Dirac fermion mode and analytical relation between fields:

One dimensional quantum mechanical system both theoretically and experimentally has studied extensively in last two decades with several successful explanation for different physical systems. There are several excellent research articles are available in the literature. Here we discuss very briefly about the Luttinger liquid physics of one-dimensional quantum many body system.

The low energy excitation of a non-interacting system. In one dimensional quantum mechanical system consists of Fermi points at $\pm k_{F}$. The low energy fermionic state thus have momentum, $k \sim \pm k_{F}$ and a single particle energy close to Fermi energy, $E_{F}$.

$$
E(k) \simeq E_{F}+\left(|k|-k_{F}\right) v_{F}+\ldots \ldots \ldots
$$

Here we consider the one-dimensional infinite length system and the elementary excitations across the Fermi points. Therefore we can write the Fermi field,

$$
\psi_{s}(x)=\int \frac{d k}{2 \pi} \psi_{s}(k) e^{i k x}
$$

Here we consider the modes of momentum expansion in a neighbourhood of $\pm k_{F}$ of width $2 \Lambda$. Therefore, we can write the fermionic field,

$\psi_{s}(x) \simeq \int_{-\Lambda}^{\Lambda} \frac{d k}{2 \pi} e^{i\left(k+k_{F}\right) x} \psi_{s}\left(k+k_{F}\right)+\int_{-\Lambda}^{\Lambda} \frac{d k}{2 \pi} e^{i\left(k-k_{F}\right) x} \psi_{s}\left(k-k_{F}\right)$

One can write this analytical expression as,

$$
\psi_{s}(x) \simeq e^{i k_{F} x} \psi_{s, R}(x)+e^{-i k_{F} x} \psi_{s, L}
$$

where $\psi_{s, R}(k)=\psi_{s}\left(k+k_{F}\right)$ and $\psi_{s, L}(k)=\psi_{s}\left(k-k_{F}\right)$. The free fermion Hamiltonian,

$$
\begin{gathered}
H_{0}=\sum_{s} \int \frac{d k}{2 \pi} \epsilon(k) \psi_{s}^{\dagger}(k) \psi_{s}(k) \\
H_{0}=\sum_{s} \int_{-\Lambda}^{\Lambda} \frac{d k}{2 \pi}\left(k v_{F}\right)\left(\psi_{s, R}^{\dagger}(k) \psi_{s, R}(k)-\psi_{s, L}^{\dagger}(k) \psi_{s, L}(k)\right)
\end{gathered}
$$

Here we use $\epsilon_{k}=k v_{F}$ near the Fermi points and $\psi_{s}^{\dagger}(x)=$ $\left(\psi_{s, R^{\dagger}}(x), \psi_{s, L}^{\dagger}(x)\right)$.

$$
\begin{gathered}
H_{0}=\sum \int \frac{d k}{2 \pi} \psi_{s}^{\dagger}(k) \sigma_{3} k v_{F} \psi_{s}(k) . \\
H_{0}=\sum_{s} \int d x \psi_{s}^{\dagger}(x) \sigma_{3} k i v_{F} \partial_{x} \psi_{s}(k),
\end{gathered}
$$

where $\sigma^{3}=\left(\begin{array}{cc}1 & 0 \\ 0 & -1\end{array}\right)$. We can define scalar field $(\phi(x)$ ) and dual field $(\theta(x))$ by the following relations $\phi(x)=\phi_{R}(x)+\phi_{L}(x), \theta(x)=-\phi_{R}(x)+\phi_{L}(x)$. One canwrite the Dirac fermion density $\rho(x)=\rho_{R}(x)+\rho_{L}(x)=$ $-\frac{1}{\sqrt{\pi}} \partial_{x} \phi_{x}$ and the corresponding current density $j(x)=v\left(\rho_{R}-\rho_{L}=\frac{v_{F}}{\sqrt{\pi}} \partial_{x} \theta(x)\right.$. The commutattion relation between the scalar field $(\phi)$ and its dual field $(\theta)$.

$[\phi(x 1), \phi(x 2)]=0=[\theta(x 1), \theta(x 2)],[\phi(x 1), \theta(x 2)]=$ $i \operatorname{sign}(x 2-x 1),[\phi(x 1), \Pi(x 2)]=i \delta(x 1-x 2)$. Where $\Pi(x)=\partial_{x} \theta(x)$. The corresponding Lagrangian debnsity for massless Dirac fermions is

$$
L=i \psi_{R}^{\dagger}\left(\partial_{t}+v_{F} \partial_{x}\right) \psi_{R}+i \psi_{L}^{\dagger}\left(\partial_{t}-v_{F} \partial_{x}\right) \psi_{L}
$$


Now we present the algebra of the operators, $e^{ \pm \phi(x)}$ and $e^{ \pm \theta(x)}$

$e^{i \alpha \sqrt{\pi} \phi(x 1)} e^{i \beta \sqrt{\pi} \theta(x 2)}=e^{i \beta \sqrt{\pi} \theta(x 2)} e^{i \alpha \sqrt{\pi} \phi(x 1)} \operatorname{sign}(x 2-x 1)$

$e^{i \alpha \sqrt{\pi} \phi(x 1)} \psi_{R / L}(x 2)=\psi_{R / L}(x 2) e^{i \alpha \alpha \sqrt{\pi} \phi(x 1)} \operatorname{sign}(x 2-x 1)$

$e^{i \beta \sqrt{\pi} \theta(x 1)} \psi_{R / L}(x 2)=-\psi_{R / L}(x 2) e^{i \beta \sqrt{\pi} \theta(x 1)} \operatorname{sign}(x 2-x 1)$

Analytical relations between order and disorder operators:

The analytical relation between the Pauli operators and $\mu$ operators are relagated to the appendix $\mathrm{B}$ of this manuscript, please put the following analytical expression in the appendix:

$$
\begin{gathered}
\mu_{z}^{2}=1=\mu_{x}^{2}, \\
\mu_{z}(n-1 / 2) \mu_{z}(n+1 / 2)=\sigma_{x}(n) . \\
\mu_{x}(n+1 / 2)=\sigma_{z}(n) \sigma_{z}(n+1), \\
\mu_{z}(n+1 / 2)=\Pi_{j=1}^{n} \sigma_{x}(j) . \\
\sigma_{z}(n)=\Pi_{j=0}^{n-1} \mu_{x}(j+1 / 2), \\
{\left[\mu_{x}(n+1 / 2), \mu_{z}\left(n^{\prime}+1 / 2\right)\right]=2 \delta_{n, n^{\prime}}} \\
{\left[\mu_{z}(n+1 / 2), \mu_{z}\left(n^{\prime}+1 / 2\right)\right]=0,} \\
{\left[\mu_{z}(n+1 / 2), \sigma_{x}\left(n^{\prime}\right)\right]=0}
\end{gathered}
$$

The operator $\mu_{z}(n+1 / 2)$ acting on the original spin of the lattice makes a spin flip of all those spin placed on the left hand side of spin at the site $\mathrm{n}$. Therefore $\mu_{z}(n+1 / 2)$ is a kink operator, it introduce the disorder in the system. It is very clear from the above analytical relation of the operators that Here we express the analytical relation between the Majorana operators and the disorder and Pauli operators:

$$
\begin{gathered}
\chi_{1}(n)=\sigma_{z}(n) \mu_{z}(n+1 / 2)=-\mu_{z}(n+1 / 2) \sigma_{z}(n) \\
\chi_{2}(n)=\sigma_{z}(n) \mu_{z}(n-1 / 2)=\mu_{z}(n-1 / 2) \sigma_{z}(n) \\
\sigma_{z}(n) \chi_{2}(n)=\mu_{z}(n-1 / 2)=\chi_{2}(n) \sigma_{z}(n)
\end{gathered}
$$

$$
\begin{gathered}
\sigma_{z}(n) \chi_{1}(n)=\mu_{z}(n+1 / 2)=-\chi_{1}(n) \sigma_{z}(n) \\
\sigma_{z}(n)=\mu_{z}(n-1 / 2) \chi_{2}(n)=\chi_{2}(n) \mu_{z}(n-1 / 2) \\
\mu_{z}(n+1 / 2) \chi_{1}(n)=-\chi_{1}(n) \mu_{z}(n+1 / 2)=\sigma_{z}(n)
\end{gathered}
$$

Derivation of Dirac equation for Majorana fermion field

The equation of motion for the $\sigma_{z}(n)$ is the following:

$$
\frac{\partial \sigma_{z}(n)}{\partial \tau}=\left[H, \sigma_{z}(n)\right]=\sigma_{x}(n) \sigma_{z}(n)
$$

The equation of motion for $\mu_{z}(n+1 / 2)$ is the following:

$$
\begin{aligned}
\frac{\partial \mu_{z}(n+1 / 2)}{\partial \tau}= & \lambda \mu_{x}(n+1 / 2) \mu_{z}(n+1 / 2) \\
& =\lambda \sigma_{z}(n) \sigma_{z}(n+1 / 2) \mu_{z}(n+1 / 2056)
\end{aligned}
$$

Now we use the properties of the $\sigma$ and $\mu$ operators to derive the equation of motion for the Majorana fields $\chi_{1}(n)$ and $\chi_{2}(n)$.

$$
\begin{aligned}
\frac{\partial \chi_{1}(n)}{d \tau}= & \frac{\partial \sigma_{z}(n)}{\partial \tau} \mu_{z}(n+1 / 2)+\sigma_{z}(n) \frac{\partial \mu_{z}(n)}{\partial \tau} \\
\frac{\partial \chi_{1}(n)}{d \tau}= & \sigma_{x}(n) \sigma_{z}(n) \mu_{z}(n+1 / 2)+\lambda \sigma_{z}(n) \sigma_{z}(n) \sigma_{z}(n+1) \mu_{z}(n+1 / 2) \\
\frac{\partial \chi_{1}(n)}{d \tau}= & -\sigma_{z}(n) \mu_{z}(n-1 / 2) \mu_{z}(n+1 / 2) \mu_{z}(n+1 / 2) \\
& +\lambda \sigma_{z}(n) \sigma_{z}(n) \sigma_{z}(n+1) \mu_{z}(n+1 / 2) \\
& \frac{\partial \chi_{1}(n)}{d \tau}=-\chi_{2}(n)+\lambda \chi_{2}(n+1)
\end{aligned}
$$

Now the equations of motion for $\chi_{2}(n)$ are

$$
\begin{gathered}
\frac{\partial \chi_{2}(n)}{d \tau}=\frac{\partial \sigma_{z}(n)}{\partial \tau} \mu_{z}(n-1 / 2) \\
+\sigma_{z}(n) \frac{\partial \mu_{z}(n-1 / 2)}{\partial \tau} \\
\frac{\partial \chi_{2}(n)}{d \tau}=\begin{aligned}
& \sigma_{x}(n) \sigma_{z}(n) \mu_{z}(n-1 / 2) \\
&+\lambda \sigma_{z}(n) \sigma_{z}(n-1) \sigma_{z}(n) \mu_{z}(n-1 / 2)(63) \\
& \frac{\partial \chi_{2}(n)}{d \tau}= \mu_{z}(n-1 / 2) \mu_{z}(n+1 / 2) \sigma_{z}(n) \mu_{z}(n-1 / 2) \\
&+ \lambda \sigma_{z}(n-1) \mu_{z}(n-1 / 2) .
\end{aligned}
\end{gathered}
$$


After a little bit of calculations and using the relation between the disorder operators (Eq. 23-30), we finally arrive the equation of motion of $\chi_{2}(n)$ as,

$$
\frac{\partial \chi_{2}(n)}{d \tau}=-\chi_{1}(n)+\lambda \chi_{1}(n-1) .
$$

These two fields, $\chi_{1}(n)$ and $\chi_{2}(n)$ satisfy the following relations, $\left\{\chi_{1}(n 1), \chi_{2}(n 2)\right\}=2 \delta_{n 1, n 2}$. One can write down the above equation in the following compact form,

$$
\left(\gamma^{0} \frac{\partial}{\partial t}+\gamma^{3} \frac{\partial}{\partial r}+m\right) \chi=0
$$

where $\chi^{\dagger}=\left(\chi_{1}, \chi_{2}\right)$ and $m=\frac{1-\lambda}{\alpha}, \gamma^{0}=\left(\begin{array}{ll}0 & 1 \\ 1 & 0\end{array}\right), \gamma^{3}=$ $\left(\begin{array}{cc}1 & 0 \\ 0 & -1\end{array}\right)$. 